\title{
Visualization of Primordial Germ Cells in Transgenic Rainbow Trout Carrying Green Fluorescent Protein Gene Driven by vasa Promoter.
}

goro YOSHIZAKI, YUTAKA TAKEUCHI, HARUo TOMINAGA, TERUMASA KOBAYASHI AND TOSHIO TAKEUCHI

Department of Aquatic Biosciences, Tokyo University of Fisheries, Minato-ku Tokyo, 108-8477, Japan (goro@tokyo-u-fish.ac.jp)

SUMMARY: To develop a cell-mediated gene transfer technique in fish, a cell line that can differentiate into the germ cell lineage is needed. In this study, we developed techniques to identify, visualize, and isolate live primordial germ cells (PGCs) for in vitro culture and later for gene transfer work. In many animals, the vasa gene products have been found in the germ cell lineage, and we have previously identified and characterized its homologue (RtVLG) in rainbow trout. Whole mount in situ hybridization of trout embryos at various developmental stages revealed that transcripts were localized to PGCs, suggesting that RtVLG transcription can be used as a marker for PGCs. To identify PGCs in vivo, we constructed a plasmid containing the green fluorescent protein (GFP) gene driven by the RtVLG regulatory regions (pvasa-GFP), microinjected it into fertilized eggs, and raised mature fish. The $F 1$ generation of these transgenic trout showed green fluorescence only in PGCs in all developmental stages. This expression pattern was consistent with that of the endogenous RtVLG gene. To obtain PGCs for in vitro culture, the genital ridges were excised from transgenic embryos, dissociated by trypsin, and a flow cytometer was used to sort them into GFP-positive and GFP-negative fractions. RT-PCR analysis revealed that RtVLG was expressed only in GFP-positive cells. These results confirm that the isolated GFP-positive cells are PGCs. We are presently attempting to establish cell lines for further work.

\section{KEY WORDS: primordial germ cell, vasa, GFP, transgenic fish, flow cytometry}

\section{INTRODUCTION}

A cell-mediated gene transfer technique would make it possible to modify endogenous gene sequences via homologous recombination. This technique is called gene targeting and will provide an especially powerful tool for breeding, for basic studies of gene expression, and for understanding the function of gene products. The cell-mediated gene transfer method follows three major steps (Fig. 1): I) the establishment of stem cell lines which can differentiate into the germ cell lineage; II) the introduction of a foreign gene into cultured cells, and selection of stably transformed cells using markers such as drug resistance; and III) the conversion of the transformed cells to individual fish via germ-line chimera fish production. The germ-line chimera is produced by transplanting the transformed cells into developing embryos. The fish that hatch consist of 2 types of cells - those derived from the host egg, and those from the transformed cells. If the transplanted cells can differentiate into the germ cell lineage, the gonads of chimeric fish can contain gametes from the transplanted cells. This means that if eggs and sperm are both from the transplanted cells, the resulting fish will be derived completely from the transformed cells.

In mice, cells containing a genome modified by homologous recombination can be selected in vitro by using marker genes ${ }^{2}$. If the parent cells are totipotent cells, such as embryonic stem (ES) cells, the genetic modifications will appear in the germ cell lineage in chimeric individuals ${ }^{3)}$. In mice, ES 
cell lines that can differentiate into the germ cell lineage have been established ${ }^{4}$ but in fish, the lack of totipotent cell lines or a cell line that can

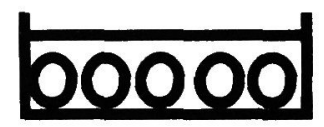

I) Establishment of a stem cell line that can differentiate into germ cells

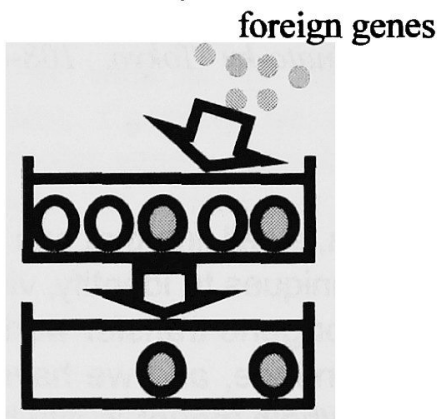

II) Gene transfer and selection of transformed cells in vitro

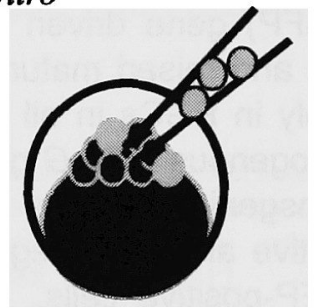

III) Transplantation of transformed cells into host eggs (germ-line chimera production)
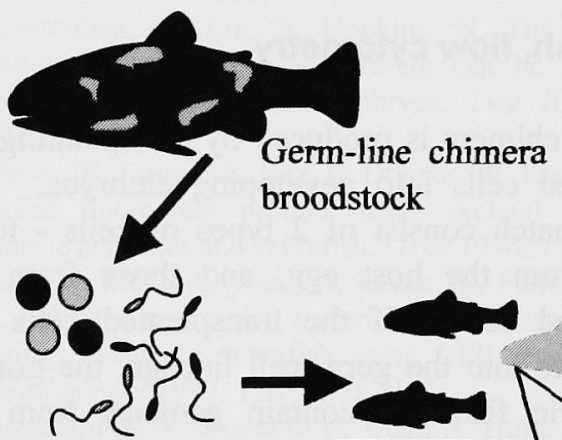

Gametes derived from chimera Transgenic offspring

Fig. 1 Schematic representation of the strategy to produce transgenic fish using "cell-mediated gene trasnsfer". White and gray cells show non-transformed and transformed cells, respectively. Black and gray fishes (or gametes) represent fishes (or gametes) derived from the host eggs and transplanted cultured cells carrying foreign genes, respectively ${ }^{1)}$. differentiate into germ cell lineage is a major obstacle to achieving the cell-mediated gene transfer. Although there have been many attempts to establish ES-like cells from early blastomeres ${ }^{5,6}$, germ line-competent ES cell lines have not yet been established. Therefore, we have chosen the primordial germ cell (PGC), a precursor of germ cell lineage, as the initial material for in vitro cell culture used for the cell-mediated gene transfer. This is because the most important characteristic required for the cells used is the ability to differentiate into the germ cell lineage.

In order to establish a cell line from PGCs, techniques to identify and isolate live PGCs are necessary. In fish, however, PGCs cannot be distinguished by morphological characteristics in live embryos, and a convenient molecular marker has not yet been reported. Vasa gene products have been found in the germ cells of a wide range of animals including zebrafish ${ }^{7,8,9,10}$. Therefore, in this study, isolation and characterization of a rainbow trout (Oncorhynchus mykiss) vasa cDNA was carried out as a first step to examine the applicability of vasa transcripts as a marker of PGCs. Then, we have cloned and characterized the rainbow trout vasa-like gene (RtVLG) regulatory regions, and produced transgenic trout carrying a green fluoresence protein (GFP) reporter gene driven by the RtVLG regulatory regions in order to visualize live PGCs in vivo. Finally, a method to isolate live primordial germ cells was established.

\section{ISOLATION AND CHARACTERIZATION OF RAINBOW TROUT VASA-LIKE GENE CDNA ${ }^{11)}$}

Cloning of vasa cDNA was performed by degenerate- and RACE-PCR. The predicted amino acid sequence contained eight consensus sequences for the DEAD protein family and five arginineglycine-glycine repeats, a common character of Vasa homologues ${ }^{12}$. Overall the amino acid sequence was $79.2 \%$ similar to the Vasa protein of Drosophila 13). Whole mount in situ hybridization of embryos (14-days post fertilization, eyed stage -24 days; and hatching stage -32 days) revealed that transcripts were localized to the putative PGCs (Fig. 2). In adult rainbow trout, both ovaries and testes contained large amounts of vasa transcripts. Reverse transcription-PCR analysis of unfertilized eggs showed that vasa is a maternal factor. Although we 
have not determined whether vasa functions as a germ cell determinant, its restricted expression in the germ cell lineage suggested that it can be used as a marker for PGCs. This marker will make it possible to identify the PGCs or presumptive PGCs in early trout embryos whose germ cells can not be distinguished by morphological characteristics.

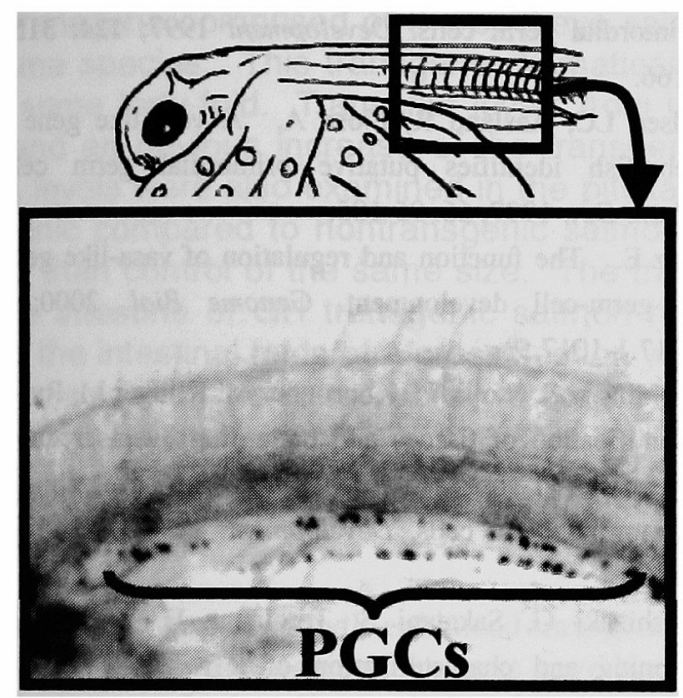

Fig. 2 In situ localization of RtVLG transcripts in an eyedstage embryo. Whole mount in situ hybridization was performed with an anti-sense DIG-labeled cRNA probe. RtVLG transcripts were localized in PGCs ${ }^{11)}$.

\section{PRODUCTION OF TRANSGENIC RAINBOW TROUT CARRYING A GFP GENE DRIVEN BY THE RtVLG PROMOTER ${ }^{14)}$}

Since the vasa transcript is restricted to the germ cell lineage, its regulatory regions should be activated only in PGCs. Therefore, we produced transgenic trout carrying GFP gene driven by the RtVLG regulatory regions (pvasa-GFP, Fig.3) in order to

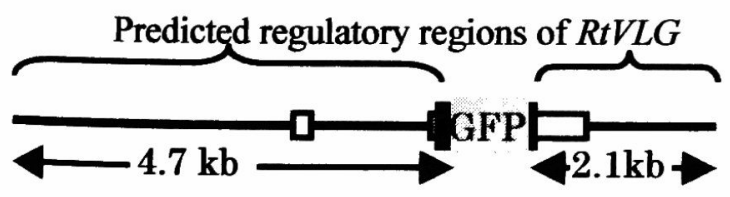

Fig.3 Structure of pvasa-GFP. White and black boxes represent untranslated regions and amino acid coding regions, respectively.

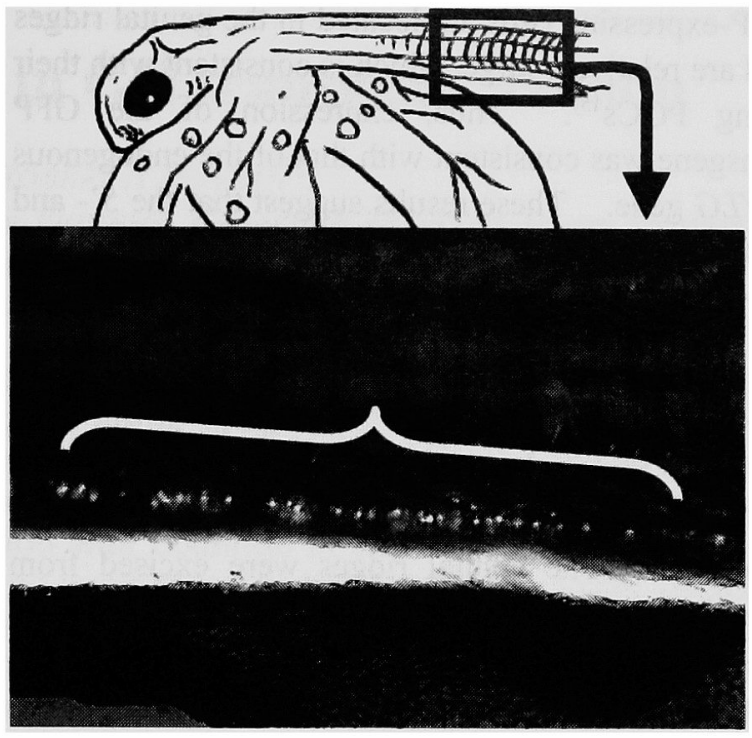

Fig.4 Fluorescence picture of the trunk region from an F1 transgenic embryo carrying pvasa-GFP. All PGCs showed green fluorescence.

visualize live PGCs in vivo. Using PCR with primers specific for the first and second exons and two consecutive vectorette-PCRs for the 5'-flanking region, we amplified a 4.7-kb DNA fragment containing $3.3 \mathrm{~kb}$ of the 5'-flanking region, $130 \mathrm{bp}$ of the first exon, $1.3 \mathrm{~kb}$ of the first intron, and a part of the second exon including the start codon. The 3'vectorette-PCR amplified $1.5 \mathrm{~kb}$ of the 3'-flanking region. The plasmid DNA for microinjection, designated as pvasa-GFP, was constructed using the $4.7-\mathrm{kb} 5$ '-fragment, the enhanced green fluorescent protein (EGFP) gene, the 3'-untranslated region ( 0.6 $\mathrm{kb})$ derived from $R t V L G$ cDNA ${ }^{11)}$ and $1.5-\mathrm{kb}$ of the 3'-flanking region. This construct was microinjected into the cytoplasm of fertilized eggs. In the transgenic trout, cells showing green fluorescence were first observed at the mid-blastula stage ( 2.5 days after fertilization), but expression was not restricted to a particular location, or to a particular cell type. However, at the hatching stage, $69.2 \%$ of the transgenic embryos showed GFP gene expression only in PGCs, an expression pattern consistent with that obtained by whole-mount in situ hybridization with an antisense $R t V L G$ cRNA probe. The F1 and F2 embryos produced by mating wildtype females and transgenic males showed green fluorescence only in PGCs during all developmental stages (Fig.4). An immunohistochemical study of hatching-stage transgenic trout revealed that the 
GFP-expressing cells are located in the genital ridges and are relatively large, which is consistent with their being PGCs ${ }^{15}$. Thus, expression of the GFP transgene was consistent with that of the endogenous RtVLG gene. These results suggest that the 5'- and 3'-flanking regions and the first intron had enough cis-elements for PGC-specific expression.

\section{isolation Of LIVE PGCs USING A FLOW CYTOMETER}

GFP-labeled PGCs were purified using a flowcytometer. The genital ridges were excised from hatching embryos using green fluorescence as an indicator. The isolated genital ridges were dissociated by $0.5 \%$ trypsin and sorted into GFPpositive cells and GFP-negative cells. RT-PCR analysis with RtVLG-specific primers confirmed that RtVLG was expressed only in GFP-positive cells, confirming that they were PGCs.

In this study, we developed techniques to visualize and isolate live PGCs and now culturing these for gene transfer experiments. We have previously developed a method to transplant blastomeres into early embryos ${ }^{16)}$, and are currently adapting this for transfer of PGCs into embryos, to obtain functional gametes derived from isolated PGCs.

\section{ACKNOWLEDGEMENTS}

This study was supported by grants from the Minstry of Agriculture, Forestry and Fisheries (Bio Design Project, BDP-01-IV-2-8) and Japan Society for the Promotion of Science (JSPS-RFTF97L00902).

\section{REFERENCE}

1. Yoshizaki G. Gene transfer in salmonidae: Applications to aquaculture. Suisanzoshoku 2001; 49: 137-142.

2. Thomas $\mathbf{K}$. Targeted mutagenesis in embryo-derived stem cells. In: First N, Haseltine, FP (eds). Transgenic Animals. Butterworth-Heinemann, Boston. 1991; 45-54.

3. Nagy A. Formation of mouse chimeric embryos from ES cells. In: Houdebine LM (ed). Transgenic Animals, Generation and Use. Harwood Academic Publishers, Amsterdam. 1997; 167-172.

4. Flechon JE. What are ES cells? In: Houdebine LM (ed). Transgenic Animals, Generation and Use. Harwood Academic Publishers, Amsterdam. 1997; 157-166.

5. Hong Y, Chen S, Schartl M. Embryonic stem cells in fish: current status and perspectives. Fish Phys. Biochem.
2000; 22: 165-170.

6. Hong $\mathrm{Y}$, Chen S, Winkler C, Schartl M. Medakafish embryonic stem cells as a model for genetic improvement of aquaculture livestocks. In: Gal L, Halvorson (eds). New Developments in Marine Biotechnology. Plenum Press, New York. 1998; 129-134.

7. Yoon C, Kawakami K, Hopkins N. Zebrafish vasa homologue RNA is localized to the cleavage planes of 2 and 4-cell-stage embryos and is expressed in the primordial germ cells. Development 1997; 124: 31573166.

8. Olsen LC, Aasland R, Fjose A. A vasa-like gene in zebrafish identifies putative primordial germ cells. Mech. Dev. 1997; 66: 95-105.

9. Raz $E$. The function and regulation of vasa-like genes in germ-cell development. Genome Biol. 2000; 1: 1017.1-1017.5.

10. Weidinger $G$, Wolke $U$, Koprunner $M$, Klinger M, Raz E. Identification of tissues and patterning events required for distinct steps in early migration of zebrafish primordial germ cells. Development. 1999; 126: 52955307.

11. Yoshizaki G, Sakatani S, Tominaga H, Takeuchi T. Cloning and characterization of a vasa-like gene in rainbow trout and its expression in the germ cell lineage. Mol. Reprod. Develop. 2000; 55: 364-371.

12. Braat AK, Speksnijder JE, Zivkovic D. Germ line development in fishes. Int. J. Dev. Biol. 1999; 43: 745760.

13. Hay B, Jan LY, Jan YN. A protein component of Drosophila polar granules is encoded by vasa and has extensive sequence similarity to ATP-dependent helicase. Cell 1988; 55: 577-587.

14. Yoshizaki G, Takeuchi Y, Sakatani S, Takeuchi T. Germ cell-specific expression of green fluorescent protein in transgenic rainbow trout under control of the rainbow trout vasa-like gene promoter. Int. J. Dev. Biol. 2000; 44: 323-326.

15. Patino T, Takashima F. Gonads. In: Takashima F, Hibiya T (eds). An Atlas of Fish Histology: Normal and Pathological features. $2^{\text {nd }}$ edition. Kodansha-Gustav Fischer Verlag, Tokyo. 1995; 128-153.

16. Takeuchi Y, Yoshizaki G, Takeuchi T. Production of germ-line chimeras in rainbow trout by blastomere transplantation. Mol. Reprod. Dev. 2001; 59: 380-389. 